\title{
Fruiting Myxobacters as Viewed with a Scanning Electron Microscope
}

ELLIS R. BROCKMAN and ROBERT L. TODD

\author{
Central Michigan University, Mt. Pleasant, Michigan 48858 and University of Georgia, \\ Athens, Georgia 30601
}

\begin{abstract}
Unfixed fruiting bodies of myxobacters have been viewed in great detail with a scanning electron microscope. Specimens of species of the genera Myxococcus, Chondrococcus, Archangium, Stelangium, Melittangium, Cystobacter, Polyangium, Stigmatella, and Chondromyces were examined. The desirability of using the scanning electron microscope for the study of the gross morphology of myxobacter structures has been clearly demonstrated.
\end{abstract}

The fruiting myxobacters possess a life cycle of three phases: the vegetative growth phase, in which the bacilli grow and reproduce by binary-transverse fission; the aggregative phase, in which vegetative cells in a localized area move toward a central point to initiate the formation of a fruiting body; and last, the fruiting-body phase, a period that results in the establishment of a mature fruiting body. The fruiting body contains or bears the resting cells, microcysts, or myxospores and has a morphology that is typical for the given species. Myxobacters are usually identified by the form and structure of the mature fruiting body. In the past the gross morphology of fruiting bodies has been illustrated primarily by three techniques: a brief chronological listing of these includes line drawings by Thaxter (9), lithographs by Jahn (2), and photomicrographs obtained by compound microscopy (McCurdy [3] and Smit and Clark [8]).

Scanning electron microscopy has only recently been employed in the study of myxobacter fruiting bodies. I. L. Roth (Bacteriol. Proc., p. 21, 1971) reported the observation of a colony of a Myxococcus species. Unpublished studies by P. Hogan and I. L. Roth (ASM News, 37:53, 1971) and P. L. Grilione (personal communication) included fruiting bodies of Myxococcus species. McNeil and Skerman (7) reported the observation of a number of myxobacters by scanning electron microscopy. The present scanning electron microscopy study (presented in part at the 72 nd Annual Meeting of the American Society for Microbiology, Philadelphia, Pa., April 1972) was undertaken to expand on the documentation of the fine detail of the fruiting bodies of myxobacters.

\section{MATERIALS AND METHODS}

Myxobacter specimens. The specimens of fruiting myxobacters were obtained from the herbarium collection of one of us (E.R.B.). The fruiting bodies were on various substrates, either bark, rabbit pellets, filter paper, or membrane filter, and most were at least 10 years old. All of the isolates were identified according to Bergey's Manual (1) and the studies of McCurdy (4-6). The Myxococcus fulvus specimen was from a rabbit pellet culture isolated from South Carolina soil. The Myxococcus xanthus and Chondromyces crocatus specimens were also from rabbit pellet cultures, and both had been isolated from soil collected in Missouri. The specimen of Myxococcus stipitatus, isolated from a Michigan stream, was on a membrane filter. Chondrococcus coralloides no. 1 and the Cystobacter fuscus specimen were from rabbit pellet cultures isolated from St. John, U.S.V.I. soil. $C$. coralloides no. 2 and the Archangium primigenium and Melittangium lichenicolum specimens were from Ulmus americana bark collected in Missouri. The specimen of Stelangium muscorum was from Juglans nigra bark collected in Missouri. The Polyangium vitellinum and Stigmatella aurantiaca specimens were both from Liriodendron tulipifera bark collected in South Carolina. The specimen of Polyangium cellulosum was from a filter-paper culture isolated from Brazilian soil.

Specimen preparation. A small piece of the air-dried substrate bearing the fruiting bodies was attached to the specimen stub with double-adhesive cellulose tape and was metal coated at $10^{-3}$ torr with palladiumgold to a uniform thickness of 100 to $200 \mathrm{~nm}$. A Cambridge Stereoscan electron microscope (Mark IIA, Cambridge Instruments Co. Ltd., London, England) was used. With the microscope operating at an accelerating voltage of $10 \mathrm{kV}$, the electrons were allowed to strike the stage at an angle of 20 to $30^{\circ}$. The resulting images were recorded with sheet film (type $55 \mathrm{P} / \mathrm{N}$; Polaroid Corporation, Cambridge, Mass.). 


\section{RESULTS AND DISCUSSION}

The surface of the fruiting body of the $M$. fulvus specimen (Fig. 1) appeared quite smooth, as has previously been illustrated (7). At the bottom of the fruiting body a constricted slime base was revealed. In culture, this base or attachment site was often more pronounced, and the fruiting bodies were often elevated by a rudimentary sporangiophore.

The fruiting body of the $M$. xanthus speci- men (Fig. 2) had a rough-textured surface. In addition, the fruiting body appeared to have deliquesced and was attached to the substrate by a broad base. This view of $M$. xanthus was not completely typical, however, for young cultures possessed fruiting bodies similar in shape to those of $M$. fulvus (Fig. 1).

The fruiting body of the $M$. stipitatus specimen (Fig. 3,4) was a spherical sporangium supported on a well-developed slime sporangiophore. The surface of the sporangium was
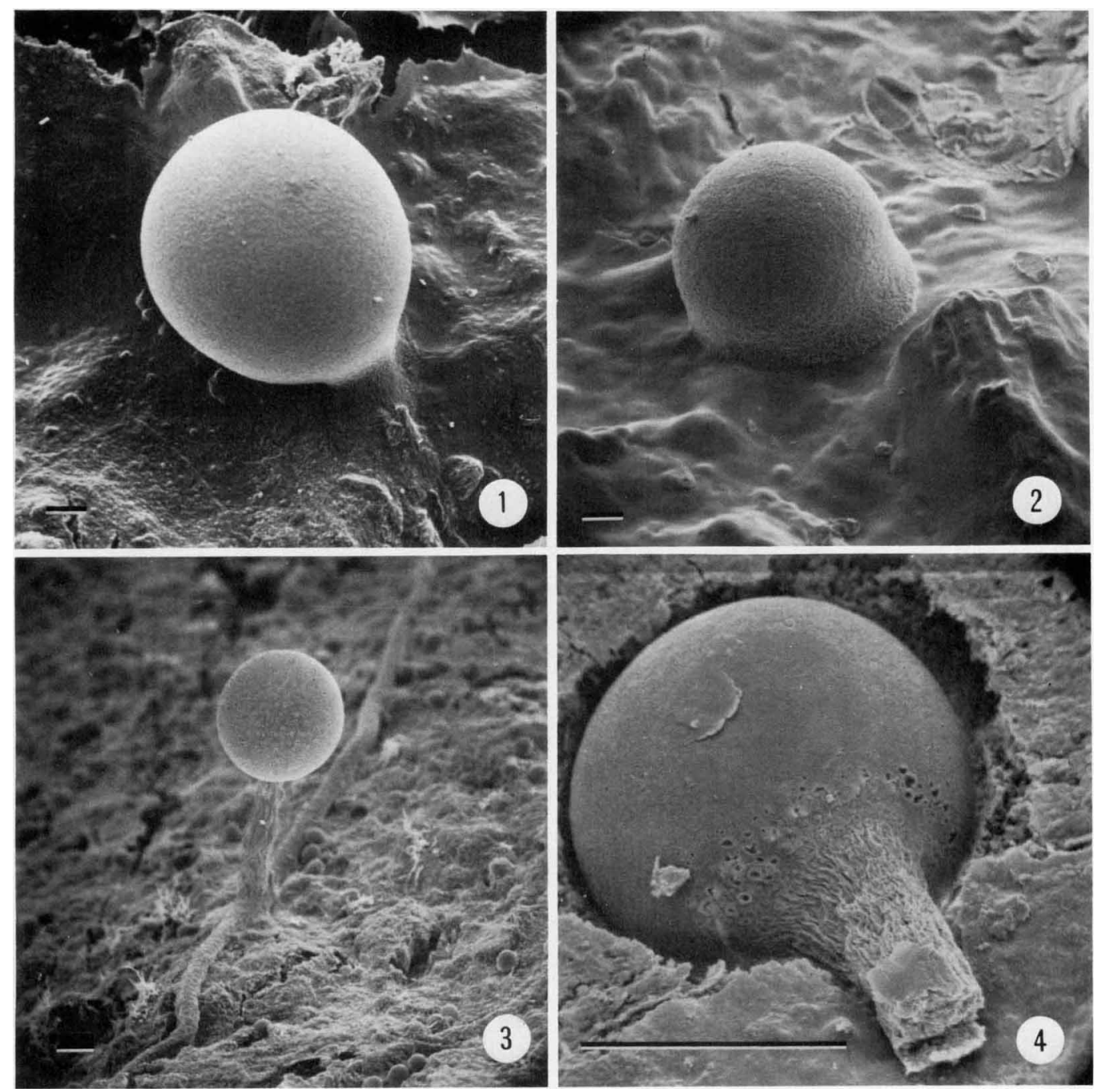

FIG. 1. M. fulvus. Fruiting body on a rabbit pellet. $\times 270$. Marker, $20 \mu \mathrm{m}$.

FIG. 2.M. xanthus. Fruiting body on a rabbit pellet. $\times 255$. Marker, $20 \mu \mathrm{m}$.

FIG. 3. M. stipitatus. Fruiting body on a membrane filter. The fructification occurred on a fiber that was also isolated from the water. $\times 240$. Marker, $20 \mu \mathrm{m}$.

FIG. 4. M. stipitatus. Fruiting body on a membrane filter. The vacuum treatment may have displaced the fruiting body. $\times 1,620$. Marker, $20 \mu \mathrm{m}$. 
pitted. This may have been due to the vacuum treatment employed for specimen preparation. In any case, the pitting demonstrated a partial collapse of the myxospores and slime of the sporangium. The wrinkled nature of the sporangiophore, unobserved in live culture, may also have been induced by the sample preparation method.

Fruiting bodies of the $C$. coralloides specimen exhibited two morphological forms: finger-like protuberances (Fig. 5) and simple columnar forms (Fig. 6). As previously noted
(7), the surfaces of these fruiting bodies appeared rough. We found the surfaces of the fruiting bodies of the $C$. coralloides specimen to be similar to those of the fruiting bodies of $M$. xanthus. McNeil and Skerman (7) made a similar observation between specimens of $C$. coralloides and Myxococcus virescens, a species often difficult to distinguish from $M$. xanthus.

The fruiting body of the $A$. primigenium specimen (Fig. 7) was flattened with an irregular, swollen surface. Although damaged, the fruiting body had a few interesting marks or
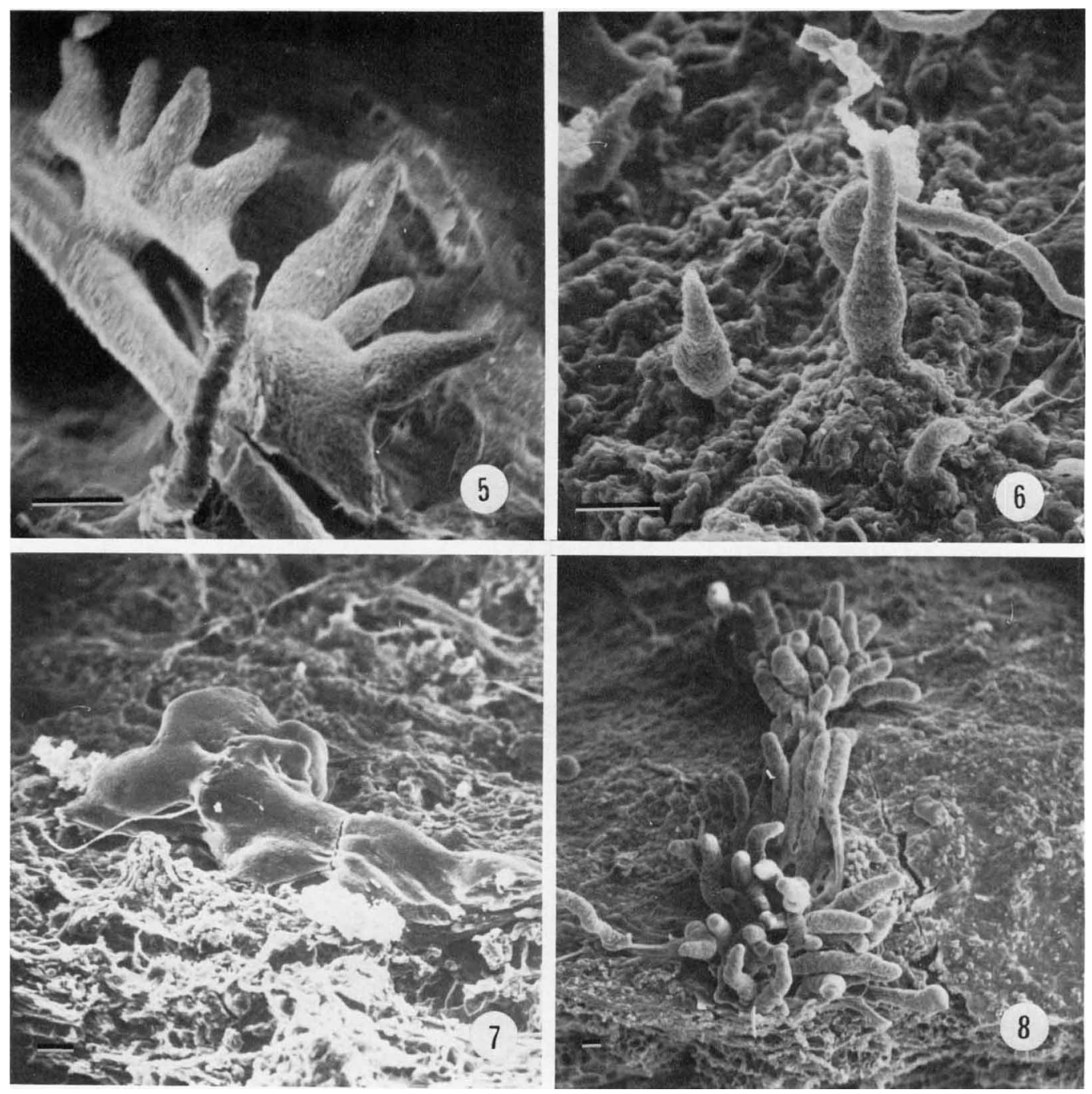

FIG. 5. C. coralloides no. 1. Fruiting bodies with finger-like projections. The structures formed on a hair that was on the rabbit pellet surface. $\times 580$. Marker, $20 \mu \mathrm{m}$.

FIG. 6. C. coralloides no. 2. Columnar-type fruiting bodies on bark. $\times 560$. Marker, $20 \mu \mathrm{m}$

FIG. 7. A. primigenium. Fruiting body on bark. The crack may have resulted from the vacuum treatment. $\times 233$. Marker, $20 \mu \mathrm{m}$.

FIG. 8. S. muscorum. Cluster of fruiting bodies on bark. X128. Marker, $20 \mu \mathrm{m}$. 
scars on its surface. The origin of these marks is unknown, although they could have come from an insect. McNeil and Skerman (7) did not present a picture of this fruiting body, although they did illustrate the surface of one specimen as being quite rough.

In Bergey's Manual (1), the fruiting body of S. muscorum is described as "simple, upright, elongate, and compact or slender at the tip." In addition, we noted (Fig. 8) that the surfaces of the fruiting bodies, although rough in appearance, attached to each other in several places. This was probably due to the adhesive nature of slime on the outer surfaces of the finger-like fruiting bodies.
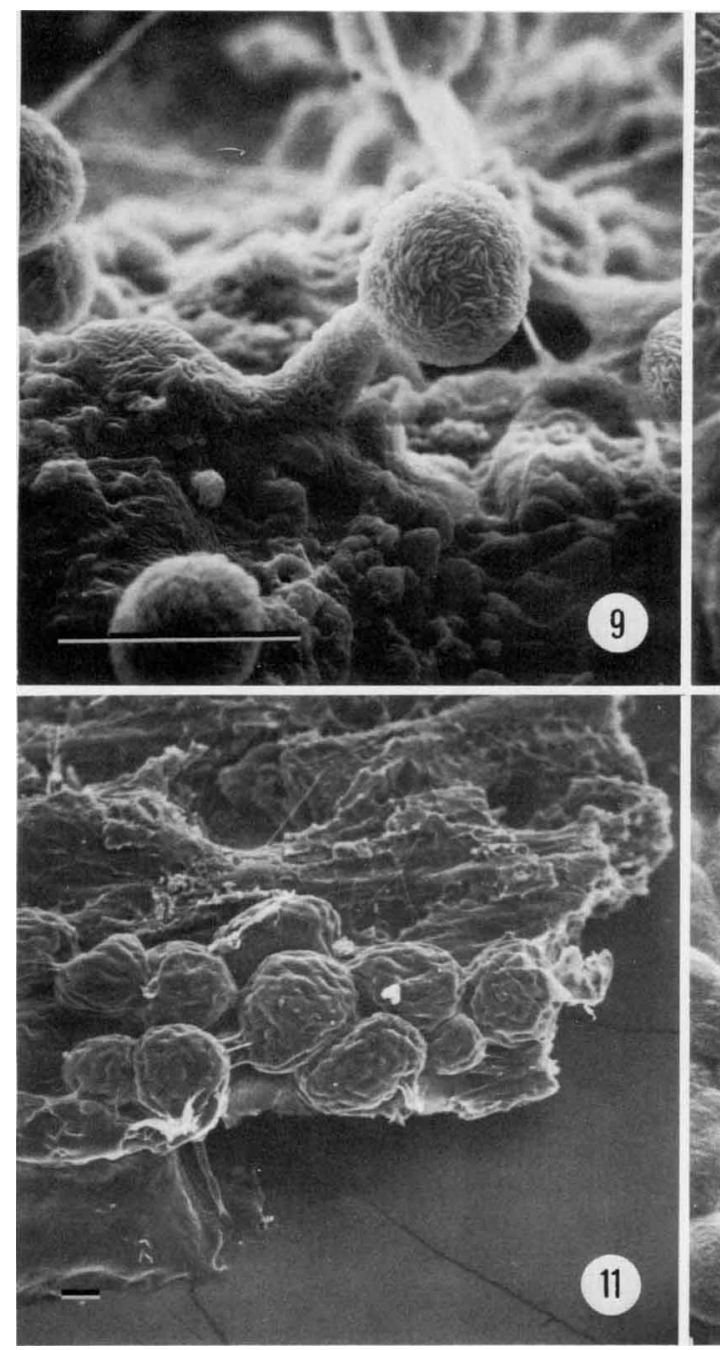

One of the most interesting fruiting bodies observed was that of the specimen of $M$. lichenicolum (Fig. 9). McNeil and Skerman (7) showed that the myxospores in the sporangium of this organism were strikingly obvious. We also noted the abundance of myxospores in the slightly tapered sporangiophore and in the swarm area at the base of this structure. From the "naked" appearance of the myxospores it was evident that $M$. lichenicalum did not produce a great amount of slime during fruiting-body formation.

The specimen of $C$. fuscus (Fig. 10) was observed to have a fruiting body that consisted of an aggregate of sporangia covered with a
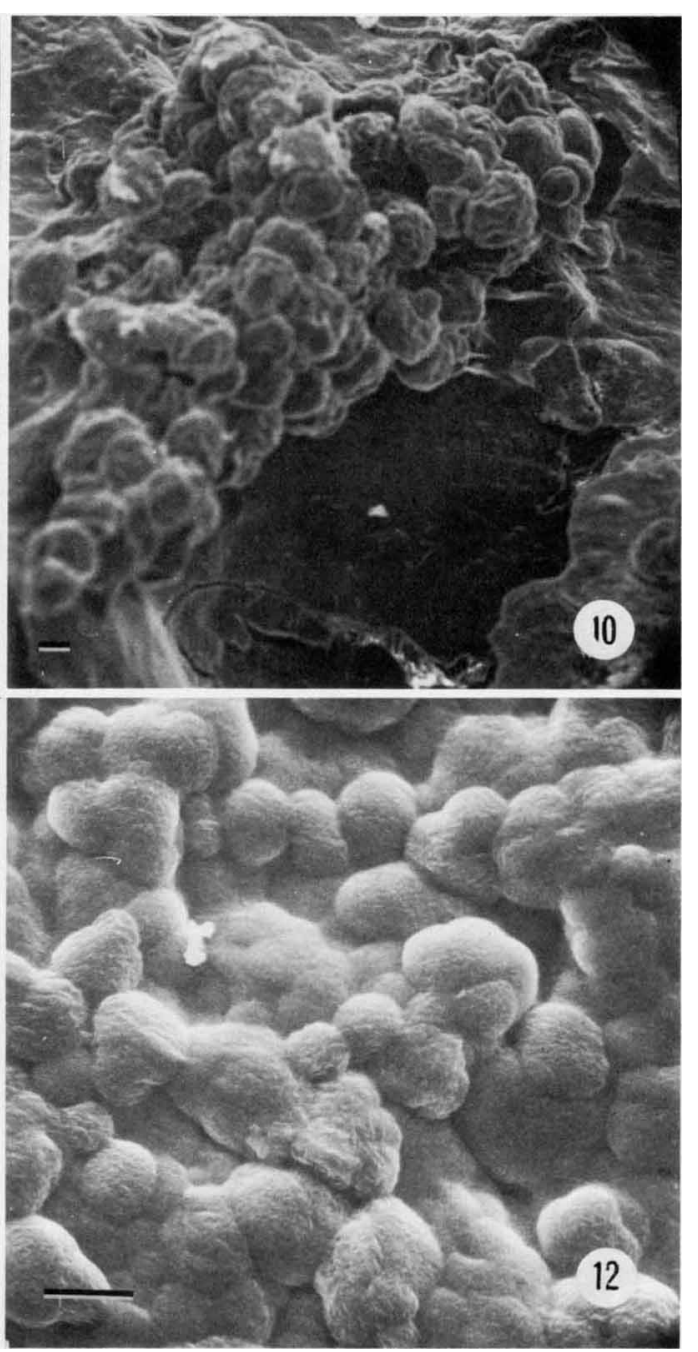

FIG. 9. M. lichenicolum. Fruiting body on bark. $\times 1$ 1,300. Marker, $20 \mu \mathrm{m}$.

FIG. 10.C. fuscus. Fruiting body on a rabbit pellet. $\times 190$. Marker, $20 \mu \mathrm{m}$.

FIG. 11. P. vitellinum. Fruiting body on bark. $\times 180$. Marker, $20 \mu \mathrm{m}$.

FIG. 12. P. cellulosum. An aggregate of fruiting bodies on filter paper. $\times 580$. Marker, $20 \mu \mathrm{m}$. 
common slime envelope. The outlines of the myxospores within the sporangia were visible through the thin sporangial wall and the delicate slime layer.

The fruiting body of the specimen of $P$. vitellinum (Fig. 11) was similar to that of the specimen of $C$. fuscus. The myxospore outlines were clearly seen through the thin sporangial walls, and the delicate nature of the slime cover was also evident. A specimen of another species of Polyangium, P. cellulosum, was observed (Fig. 12). This organism constructed fruiting bodies of numerous sporangia of various sizes. It also produced large quantities of slime in culture, and we believe that this slime, rather
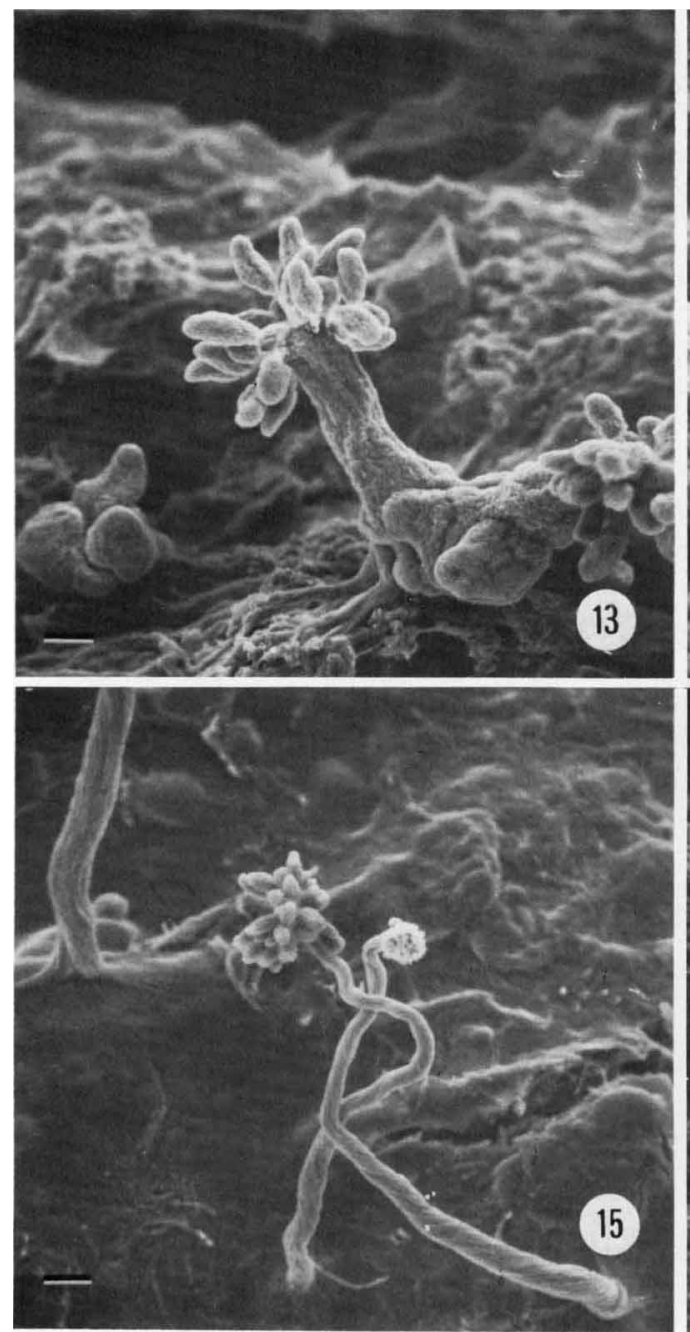

than sporangial-wall thickness, obscured the myxospore outlines.

The most complex myxobacter fruiting bodies belong to species of the genera Stigmatella and Chondromyces. A specimen of one species of each of these genera was observed: $S$. aurantiaca (Fig. 13, 14) and $C$. crocatus (Fig. 15-18). Although specimens of both species were also observed by McNeil and Skerman (7), the pictures of this study present these organisms in a different perspective. The sporangiophores in these two specimens were quite different. In the case of $C$. crocatus, they were thin, striated, and twisted. On the other hand, the specimen of $S$. aurantiaca had stout
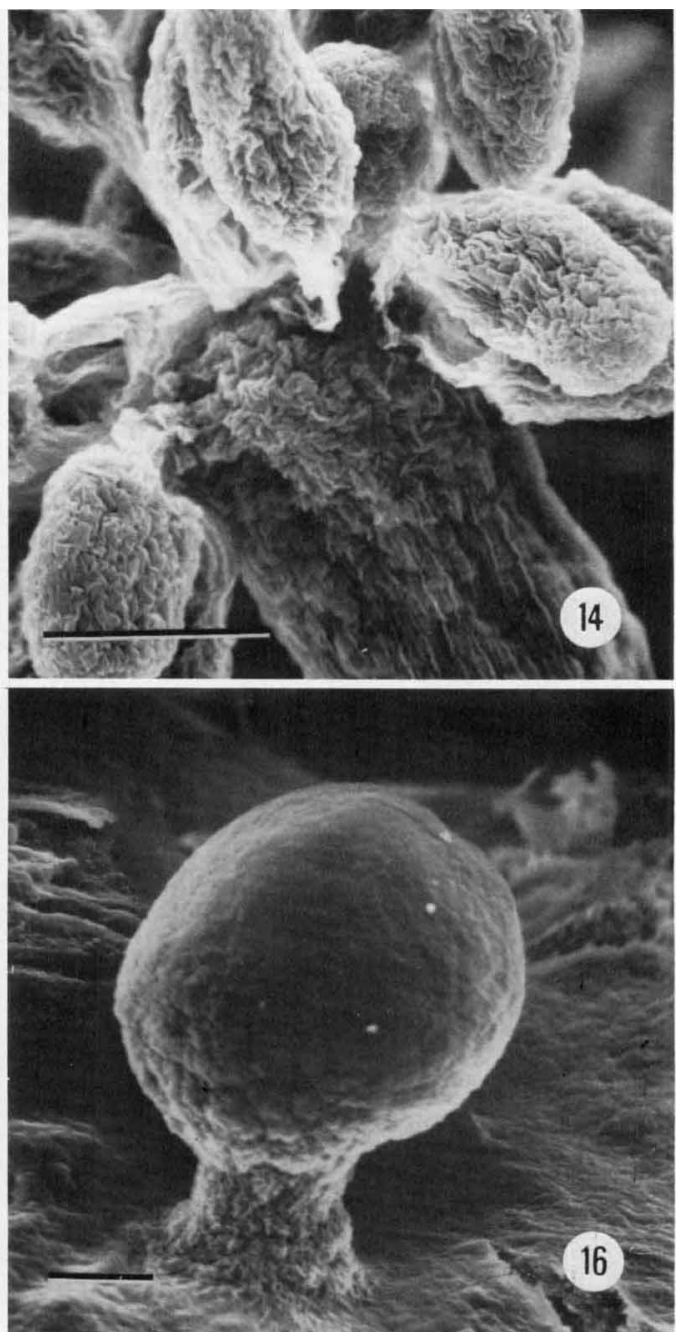

FIG. 13. S. aurantiaca. Fruiting body on bark. $\times 250$. Marker, $20 \mu \mathrm{m}$.

FIG. 14. S. aurantiaca. View of sporangia attached by pedicles to sporangiophore. $\times 1,250$. Marker, $20 \mu \mathrm{m}$.

FIG. 15. C. crocatus. Fruiting bodies on a rabbit pellet. $\times 260$. Marker, $20 \mu \mathrm{m}$.

FIG. 16. C. crocatus. Immature fruiting body on a rabbit pellet. $\times 580$. Marker, $20 \mu \mathrm{m}$. 

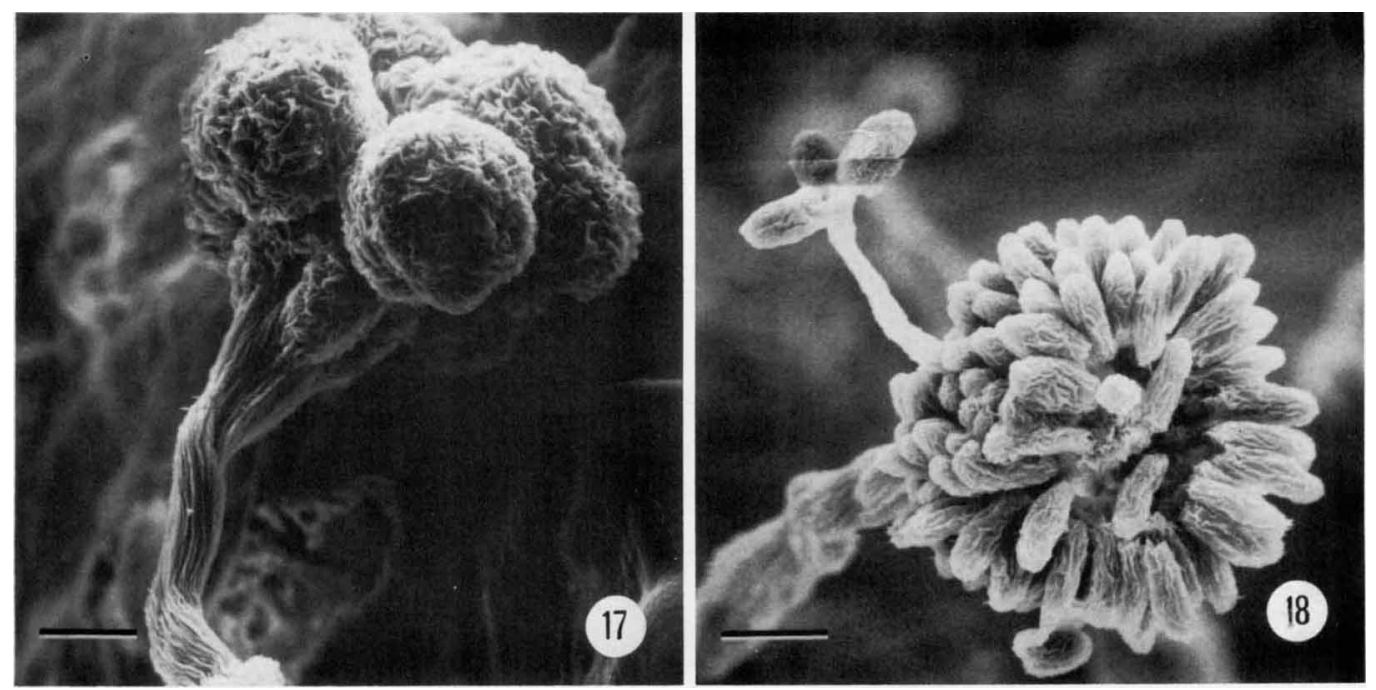

FIG. 17. C. crocatus. Immature fruiting body on a rabbit pellet. $\times 580$. Marker, $20 \mu \mathrm{m}$.

FIG. 18. C. crocatus. Sporangia of a mature fruiting body on a rabbit pellet. $\times 630$. Marker, $20 \mu \mathrm{m}$.

sporangiophores that were dense in appearance, and, upon close observation (Fig. 14), cells were noted throughout the matrix.

All of the specimens of $C$. crocatus used to demonstrate various stages in fruiting-body formation were obtained from different areas of one rabbit pellet culture. The simple, immature fruiting body (Fig. 16) resulted after the vegetative cell aggregation phase. At this point the rounded, young sporangial mass appeared smooth due to a large amount of slime production by the bacteria. The short sporangiophore was dense and obviously contained cells. At a later stage (Fig. 17), the sporangiophore was well developed, yet the sporangia had not differentiated. In the last figure (Fig. 18), the sporangia of a mature fruiting body are seen. Secondary fruiting-body formation (Fig. 18), a common occurrence in laboratory cultures of $C$. crocatus, developed when myxospores in one of the sporangia germinated and proceeded through a complete life cycle.

The scanning electron microscope serves as a most useful tool for extending scientific observation beyond the limit of the optical microscope. It will be particularly helpful in the critical examination of various stages of fruiting-body development as well as of mature myxobacter fruiting bodies.

Although partial duplication of effort exists between our work and that of McNeil and Skerman (7), this duplication is most worthwhile, for the findings of our two studies complement one another. Together they present an extensive look at the gross morphology of myxobacter fruiting bodies as viewed with the scanning electron microscope.

\section{ACKNOWLEDGMENTS}

E.R.B. thanks the Research and Creative Endeavors Committee of Central Michigan University for a travel grant that made this study possible.

We thank W. J. Humphreys and B. O. Spurlock, Electron Microscopy Laboratory, University of Georgia, Athens, for their cooperation.

\section{REPRINT REQUESTS}

Address reprint requests to: Dr. Ellis R. Brockman, Central Michigan University, Mt. Pleasant, Mich. 48858 .

\section{LITERATURE CITED}

1. Breed, R. S., E. G. D. Murray, and N. R. Smith. 1957. Bergey's manual of determinative bacteriology, 7 th ed. The Williams \& Wilkins Co., Baltimore, Md.

2. Jahn, E. 1924. Beitrage zur botanischen Protistologie. I. Die Polyangiden. Gebr. Born trager, Leipzig.

3. McCurdy, H. D. 1969. Studies on the taxonomy of the Myxobacterales. I. Record of Canadian isolates and survey of methods. Can. J. Microbiol. 15:1453-1461.

4. McCurdy, H. D., Jr. 1970. Studies on the taxonomy of the Myxobacterales. II. Polyangium and the 
demise of Sorangiaceae. Int. J. Syst. Bacteriol. 20:283-296.

5. McCurdy, H. D. 1971. Studies on the taxonomy of the Myxobacterales. III. Chondromyces and Stigmatella. Int. J. Syst. Bacteriol. 21:40-49.

6. McCurdy, H. D. 1971. Studies on the taxonomy of the Myxobacterales. IV. Melittangium. Int. J. Syst. Bacteriol. 21:50-54.

7. McNeil, K. E., and V. B. D. Skerman. 1972.
Examination of myxobacteria by scanning electron microscopy. Int. J. Syst. Bacteriol. 22:243-250.

8. Smit, M., and A. G. Clark. 1971. The observation of myxobacterial fruiting bodies. J. Appl. Bacteriol. 34: 399-401.

9. Thaxter, R. 1892. On the Myxobacteriaceae, a new order of Schizomycetes. Bot. Gaz. (Chicago) 17:389-406. 